Classification

Physics Abstracts

$61.16 \mathrm{~B}-61.10-61.72$

\title{
Strain Relaxation of Si/Ge Multilayers Investigated by Transmission Electron Microscopy and High-Resolution X-Ray Diffractometry
}

\author{
Elvio Carlino $\left({ }^{1}\right)$, Cinzia Giannini $\left({ }^{1}\right)$, Leander Tapfer $\left({ }^{1}\right)$, Kurt A. Mäder $\left({ }^{2}\right)$ and \\ Hans von $\operatorname{Känel}\left({ }^{2}\right)$ \\ ( ${ }^{1}$ ) PASTIS-Centro Nazionale Ricerca e Sviluppo Materiali (PASTIS-CNRSM) S.S. 7 Appia km. 712, \\ 72100 Brindisi, Italy \\ $\left({ }^{2}\right)$ Laboratorium für Festkörperphysics, ETH-Zürich, CH-8093, Switzerland
}

(Received March 1; accepted May 12, 1995)

\begin{abstract}
In this work we investigate the strain-relief mechanisms and the formation of structural defects of $\mathrm{Si} / \mathrm{Ge}$ multilayers grown by molecular beam epitaxy on (100)- Si substrates. The investigated specimens differ in number of periods, period thickness, and in the $\mathrm{Si} / \mathrm{Ge}$ layer thickness ratio. The structural analyses are performed by transmission electron microscopy and high-resolution X-ray diffraction. We found that a Si-Ge interdiffusion induces a broadening of the nominal thickness of the Ge layer, producing a $\mathrm{Si}_{x} \mathrm{Ge}_{1-x}$ alloy as well as a higher $\mathrm{Ge}$ content in the last periods of the multilayer structure. Our measurements suggest that the strain relaxation occurs in two steps: i) in each period of the multilayer the strain energy density is partially reduced by the formation of coherent islands; ii) at a certain value of the strain energy density, the shape of the coherent islands changes and the structures, partially or completely, relax the accumulated strain energy by nucleation of dislocations. The increase of the strain energy density is related to the measured monotonic increase of the $\mathrm{Ge}$ content as a function of the growth time.
\end{abstract}

\section{Introduction}

The $\mathrm{Si} / \mathrm{Si}_{x} \mathrm{Ge}_{1-x}(0<x<1)$ semiconductor material system attracts a very high interest for the fundamental study of the epitaxial growth of highly strained heterostructures. The main effort is focused on the understanding of the strain-relief mechanisms and of the formation of structural defects. The knowledge of these processes is fundamental for the fabrication of heterostructure devices which are based on this highly strained material system. Despite the high interest and the big effort in the last forty years, a complete comprehension of the strain relaxation mechanism in these material systems has not been achieved. Several authors report on the strain relaxation via the nucleation of misfit dislocations and on their formation mechanism [1-8]. The proposed models give a critical thickness for the relaxation by nucleation of misfit dislocations, underestimating the experimentally measured value $[9,10]$. Recently, the role of the strain energy relieved 
by creation of coherent islands has gained more and more attention [11-15]. In fact, taking into account the decreasing of the strain energy density due to the island formation, the critical thickness for the nucleation of defects can be predicted more precisely. The combination of techniques like transmission electron microscopy (TEM) and high resolution X-ray diffraction (HRXRD) allows us to characterize the crystalline structure and to determine the structural parameters with high accuracy and high spatial resolution. In the present work the strain relaxation via coherent islanding is monitored in $\mathrm{Si} / \mathrm{Si}_{x} \mathrm{Ge}_{1-x}$ multilayers. Furthermore, a monotonic increase of the $\mathrm{Ge}$ content in each multilayer period due to Ge diffusion, as a function of the growth time, has been revealed. As the Ge content increases, the island shape evolves in order to partially relax the strain energy via the nucleation of extended defects.

\section{Experimental}

The samples analyzed in the present work were grown by $\mathrm{MBE}$ at a temperature of $460{ }^{\circ} \mathrm{C}$ and at a growth rate of $0.1 \mathrm{~nm} / \mathrm{s}$. Their nominal parameters are reported in Table I.

Table I. - Nominal parameter of the analyzed specimens. $\Lambda$ is the multilayer period.

\begin{tabular}{|c|c|c|c|c|}
\hline Sample & Periods & Si-thickness (nm) & Ge-thickness (nm) & Ge-thickness/ \\
\hline$\# 230$ & 33 & 4.55 & 0.55 & 0.108 \\
\hline$\# 233$ & 21 & 6.33 & 0.83 & 0.116 \\
\hline
\end{tabular}

The specimens for TEM analyses were prepared in the cross-section geometry following the usual procedure for the mechanical and ion mill thinning. A liquid nitrogen cooled stage was used in order to minimize the generation of damage and artifacts in the specimen. TEM experiments were performed using a Philips CM 30 TEM/STEM microscope, operating at $300 \mathrm{kV}$ (interpretable resolution limit of $0.23 \mathrm{~nm}$ ) and a Jeol JEM $4000 \mathrm{EX}$ operating at $400 \mathrm{kV}$ (interpretable resolution limit of $0.16 \mathrm{~nm}$ ). As a result of the sample irradiation with $400 \mathrm{keV}$ electrons, several defects were observed. For the high resolution electron microscopy (HREM) experiments, all the samples were analyzed along the $<011>$ zone axis.

The width of each Si and Ge layer was measured from the HREM micrographs. Several effects have to be taken into account measuring the width of a strained layer from a high resolution image [16-20]; among them the effect of the crystal plane bending in the TEM thinned sample can produce a systematic error in the measurements [21-26]. This effect can be particularly severe in highly strained samples. As a consequence of the high Ge content of the sample analyzed in the present work (Tab. I), the interfaces between Ge-rich layers and Si layers should be revealed in the micrographs as an abrupt change in the image intensity. The contrast variation was measured from several HREM micrographs. In each HREM image, each pixel was represented by its height above the base plane. The width of the zone with higher Ge content was measured as the width of the image contrast variation at full width half maximum. From several measurements, the mean value of the $\mathrm{Si}_{x} \mathrm{Ge}_{1-x}$ width was calculated. As an error on the mean value the standard deviation was considered. To reduce the uncertainty in the location of the $\mathrm{Si} / \mathrm{Ge}$ interfaces we used a defocus value to maximize the contrast between the Si and Ge layers [16,27,28]. The layer width measurements reported in the next paragraph have to be considered as the average width of the zone at higher Ge content. 


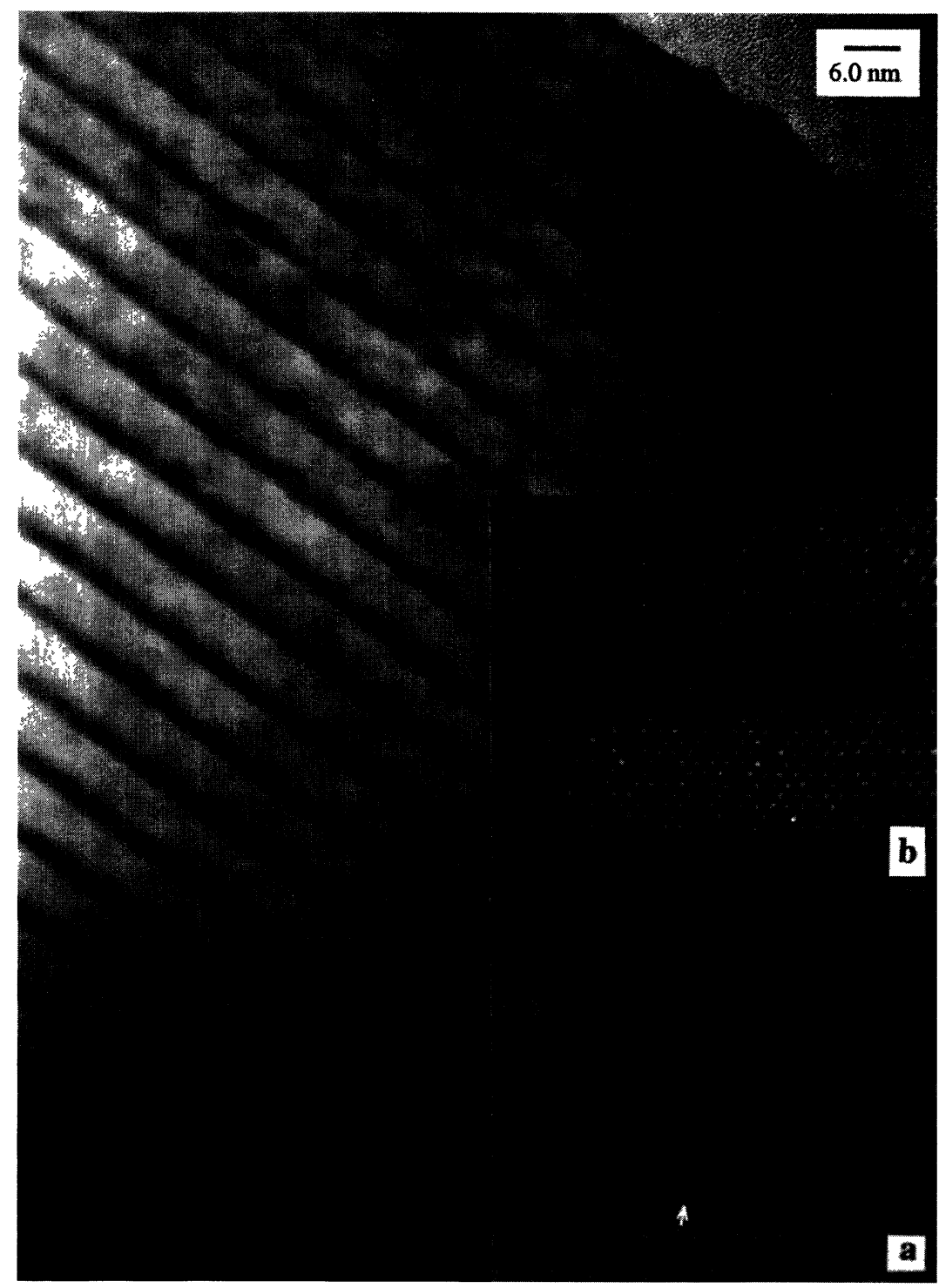

Fig. 1. - Low magnification high resolution electron microscopy micrograph in the [110] zone axis of sample $\sharp 233$; inset a):the arrow indicates an area of blurred contrast; inset b): defect free coherent island.

High-resolution X-ray diffraction measurements were performed by means of a double-crystal X-ray diffractometer (Philips, MRD) equipped with a Cu target as X-ray source $(\lambda=1.5405 \AA)$, a four-crystals symmetrical cut $\mathrm{Ge}(220)$ monochromator and a proportional counter. From these experiments we measure the average multilayer lattice parameter in the growth direction (outplane), as well as in the interface plane (in-plane), which are related to the angular distances between the zero-order superlattice satellite and the substrate peak. A simple tetragonal distortion (with respect to the Si substrate) of the epilayer unit cell has been considered for data analysis using a 2nd-order approximation of the strain function in the Takagi-Taupin equation [29]. The superlattice period $\left(\Lambda_{\mathrm{SL}}\right)$ was measured from the angular separation between the superlattice satellites peaks [30]. 


\section{Results}

Figure 1 shows a cross section of the sample $\sharp 233$. The epitaxial structure is made of a $21 \mathrm{Si}_{x} \mathrm{Ge}_{1-x}$ /Si periods; each period has an average thickness of (7.2 \pm 0.3$) \mathrm{nm}$ and is made of one $\mathrm{Si}_{x} \mathrm{Ge}_{1-x}$ layer $(1.2 \pm 0.2) \mathrm{nm}$ thick and one Si layer $(6.0 \pm 0.3) \mathrm{nm}$ thick. In some areas (Fig. 1 inset "a") the contrast of the interfaces between $\mathrm{Si}$ and $\mathrm{Si}_{x} \mathrm{Ge}_{1-x}$ layers is not sharp and this feature can be related to the intermixing of $\mathrm{Si}$ and $\mathrm{Ge}$ atoms at the interface [16,28]. The wavy aspect of the periods is due to a quasi-periodic small variation of the thickness (coherent-islands) of each $\mathrm{Si}_{x} \mathrm{Ge}_{1-x}$ period. It should be noted that the presence of this thickness variation does not create extended defects in the lattice (Fig. 1 inset "b"). This finding is in agreement with the results reported by Eaglesham et al. [15] and Le Goues et al. [31] who demonstrated that strain relaxation via Ge islanding can occur without the generation of misfit dislocations. Figure 2 shows a crosssection of a typical defect, observed in this sample, which we named "conical shaped defect". The distribution of these defects is quite regular with a density of about $1 \times 10^{8} / \mathrm{cm}^{2}$. It is a tridimensional defect that involves several periods of the epilayer and produces a relevant curvature on the surface of the film. The bending of the periods close to the defect is evident (Fig. 2). Within the defect volume a high density of stacking faults and dislocations are observed (see the arrow). The analysis of the sample in cross section allows us to detect the features of these defects in slices coming from different parts of the cone. Figure 3 shows that the number of extended defects at the "conical shaped defect" boundaries is very high (see the arrows). These extended defects are

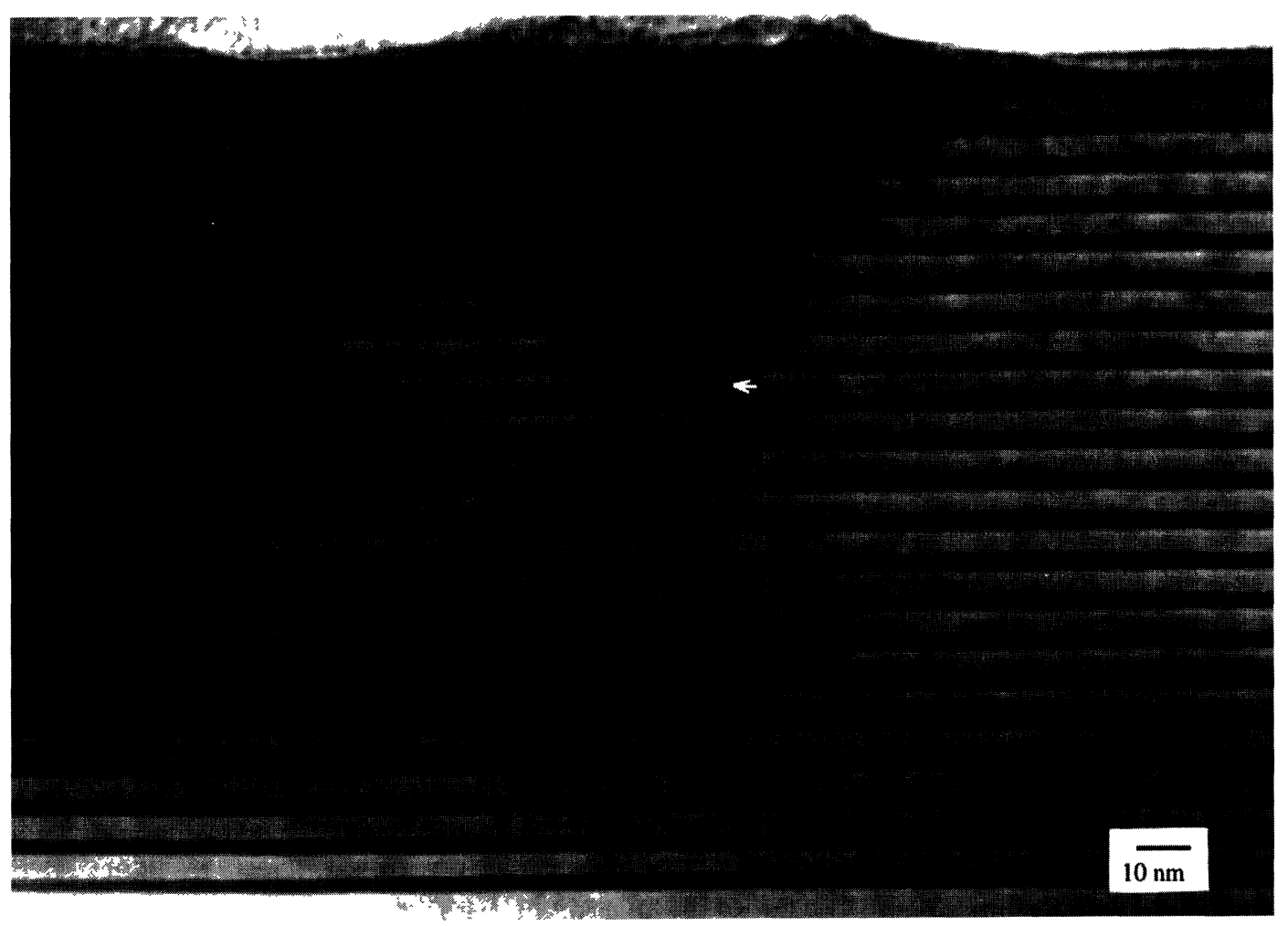

Fig. 2. - Bright field in the [110] zone axis of a "conical shaped defect" in the sample $\sharp 233$; the arrow indicates one of the several extended defects in this area. 


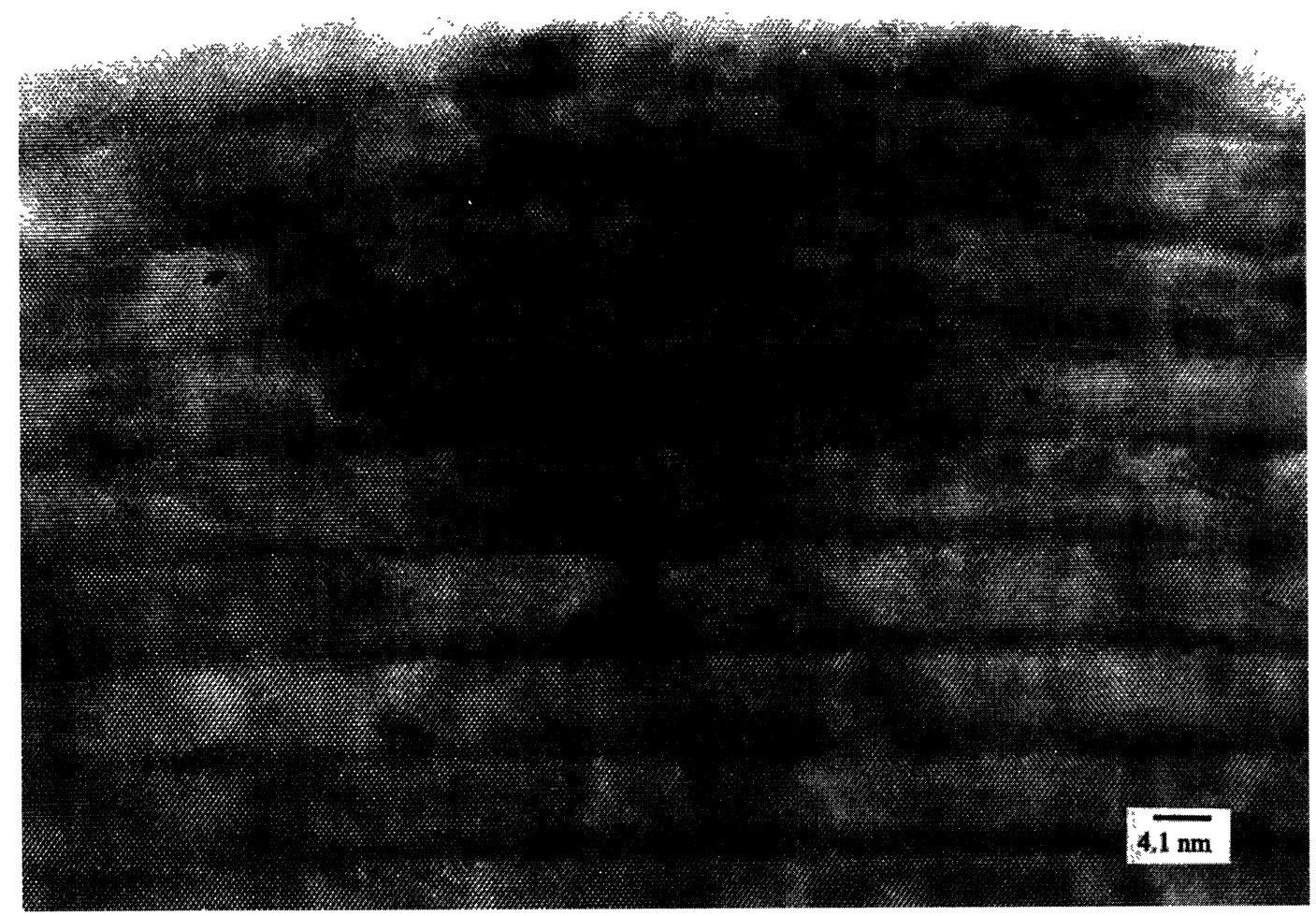

Fig. 3. - High resolution electron microscopy micrograph in [110] zone axis of a "conical shaped defect" in the sample $\sharp 233$; the arrows indicate the presence of stacking faults at the boundary of the "conical shaped defect”.

created in order to accommodate the distorted area with the undisturbed part of the epitaxial structure. In some cases the extended defects originating in the distorted volume propagate down to the substrate/epilayer interface. Figure 4 shows how the deformation starts, after few periods (see arrow $\mathrm{A}$ ) in $\mathrm{Si}_{x} \mathrm{Ge}_{1-x}$ layer. From the $8^{\text {th }}$ period a further bending in the periods is visible (see arrow $\mathrm{B}$ ) and the periodic stacking of the $\mathrm{Si}_{x} \mathrm{Ge}_{1-x} / \mathrm{Si}$ layers is lost resulting in a large area of $\mathrm{Si} / \mathrm{Ge}$ intermixing. It is worthwhile to remark that, except in the area of the "conical shaped defect", the density of extended defects in this sample is rather low.

Figure 5 shows a bright field image in $<011>$ zone axis of the 33 periods of $\mathrm{Si}_{x} \mathrm{Ge}_{1-x} / \mathrm{Si}$ of sample $\sharp 230$. Each period has an average thickness of $(5.1 \pm 0.3) \mathrm{nm}$ and is made of one $\mathrm{Si}_{x} \mathrm{Ge}_{1-x}$ layer $(1.2 \pm 0.2) \mathrm{nm}$ thick and one $\mathrm{Si}$ layer $(3.9 \pm 0.3) \mathrm{nm}$ thick. The wavy aspect of the first 16 periods of the epilayer is due to the quasi-periodic small variation of the thickness of each $\mathrm{Si}_{x} \mathrm{Ge}_{1-x}$ layer (coherent-islands). In the $17^{\text {th }}$ and $18^{\text {th }} \mathrm{Si}_{x} \mathrm{Ge}_{1-x}$ layers the waviness changes, increasing in amplitude and period (see the arrows). This latter thickness variation influences all the remaining periods and produces a waviness on the surface of the epilayer. In these areas also the density of extended defects is relatively high. It should be noted that in this case the deformation of the periods inside the "conical shaped defects" is evidently less pronounced with respect to the sample $\sharp 233$.

Figure 6 shows the high-resolution X-ray diffraction profiles recorded on the investigated samples in the vicinity of the $\mathrm{Si}(400)$ reflection. The two curves (A, B) refer to the samples $\sharp 230$ and 


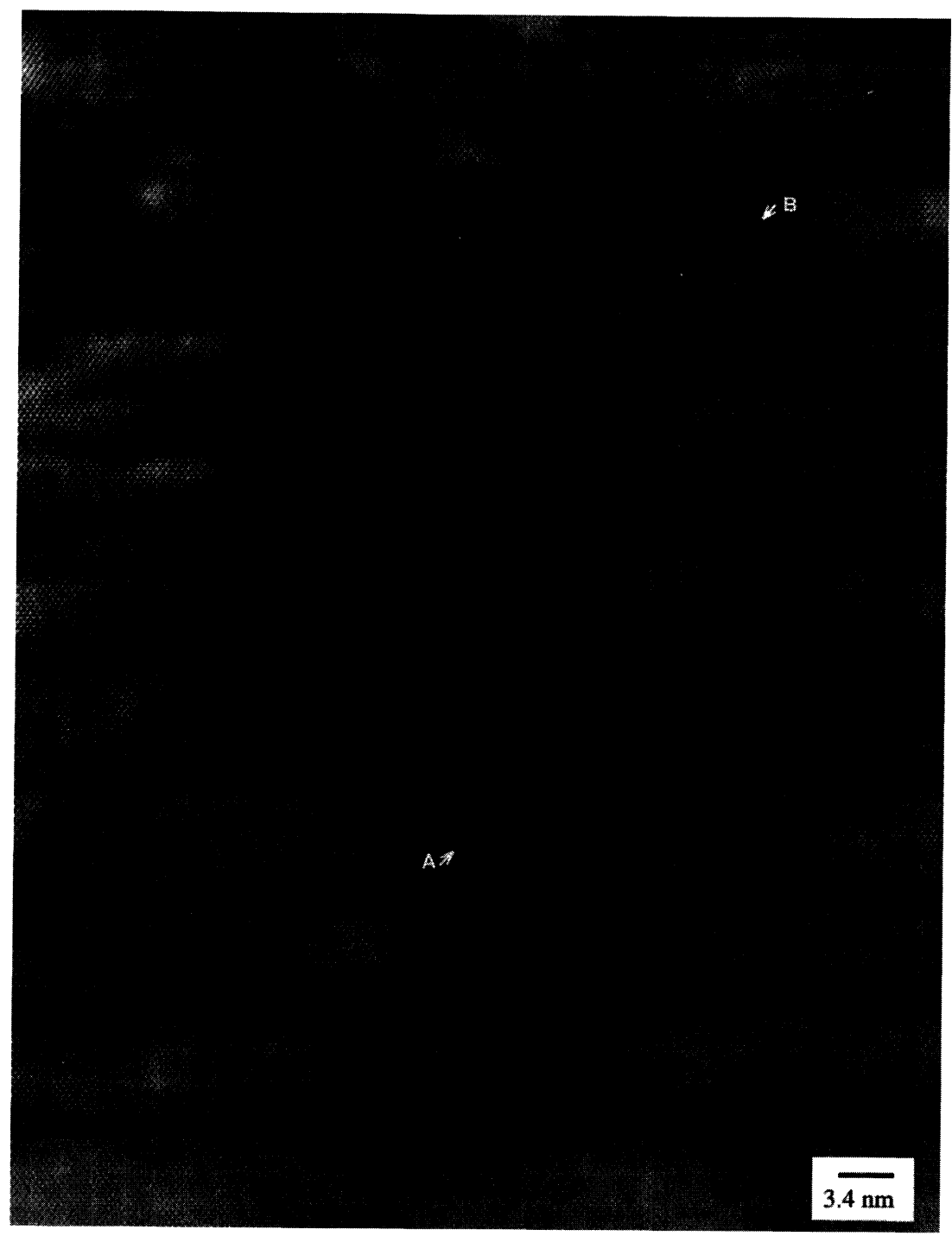

Fig. 4. - High resolution electron microscopy micrograph in [110] zone axis of a "conical shaped defect" in the sample $\sharp 233$; the arrows $A$ and B indicate respectively the area of the origin of the conical shaped defect and the bending produced by this defect in the $8^{\text {th }}$ period.

$\sharp 233$, respectively. These data, together with the asymmetric reflections data, allow us to measure the average in-plane $\left(\varepsilon_{\mathrm{i}}\right)$ and out-of-plane $\left(\varepsilon_{\mathrm{o}}\right)$ strain tensor components for the investigated multilayers (Tab. II). Table II contains also the mean superlattice period $\left(\Lambda_{\mathrm{SL}}\right)$ measured from the superlattice satellites angular distances. As can be seen from these data, samples $\sharp 230$ and $\sharp 233$ have no in-plane strain and almost the same out-plane strain value (i.e. the same average Ge content). Moreover, a strong diffuse scattering is measured on sample $\sharp 230$, especially at high angles, being the finger-print of a quite rough $\mathrm{Si}_{x} \mathrm{Ge}_{1-x} / \mathrm{Si}$ interface. This diffuse scattering slightly reduces in sample $\sharp 233$. 


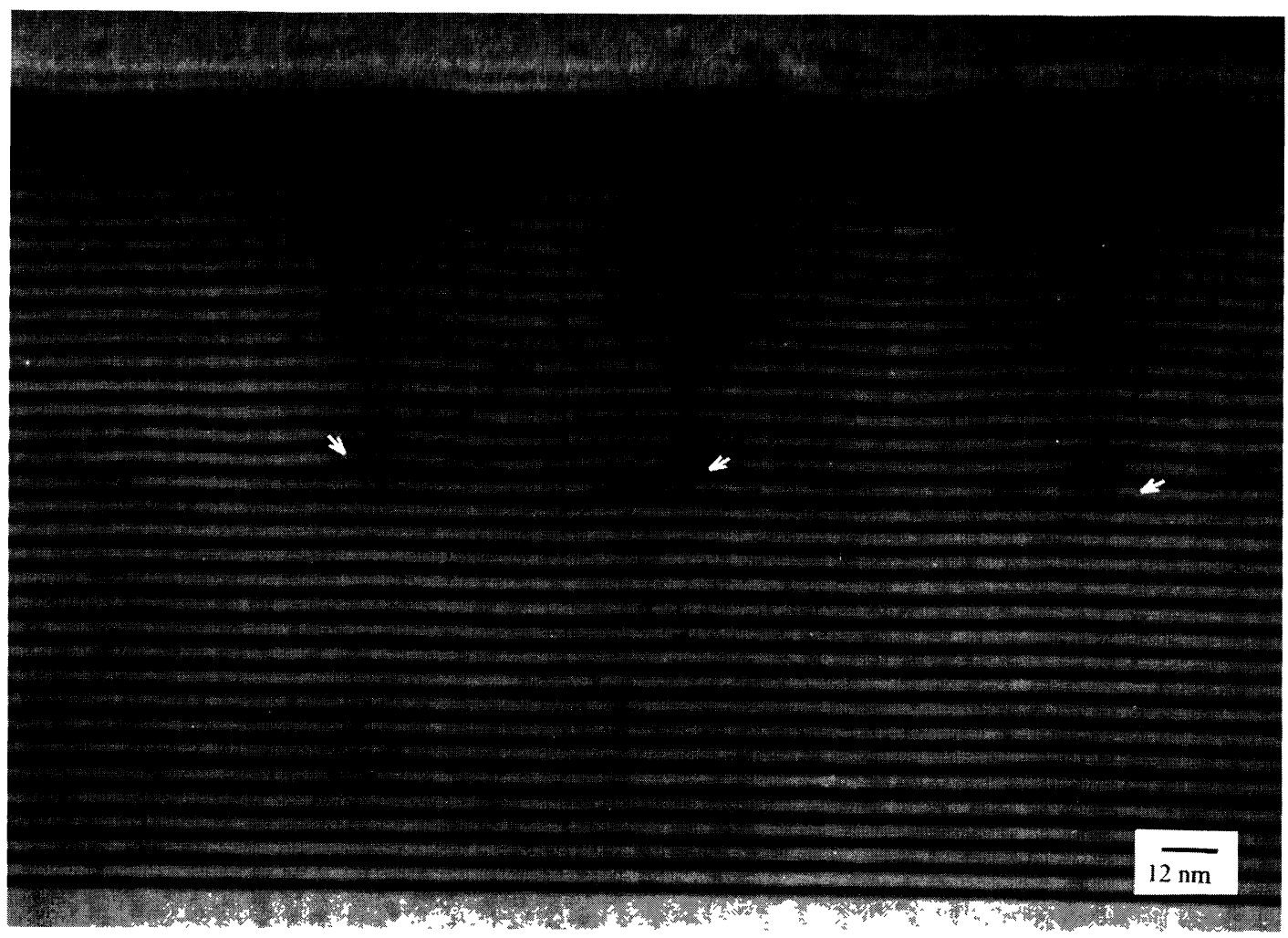

Fig. 5. - Bright field in [110] zone axis of the sample $\sharp 230$; the arrows indicate the increase of the waviness amplitude in the $17^{\text {th }}$ and $18^{\text {th }}$ periods.

Table II. - The average out-plane $\left(\varepsilon_{\mathrm{o}}\right)$, in-plane $\left(\varepsilon_{\mathrm{i}}\right)$ strain tensor components are given together with the superlattice period $\left(\Lambda_{\mathrm{SL}}\right)$.

\begin{tabular}{|c|c|c|c|}
\hline Sample & $\varepsilon_{\mathrm{o}}\left(10^{-3}\right)$ & $\varepsilon_{\mathrm{i}}\left(10^{-5}\right)$ & $\Lambda_{\mathrm{S}}(\mathrm{nm})$ \\
\hline$\# 230$ & 8.57 & 0 & 5.0 \\
\hline$\# 233$ & 8.35 & 0 & 7.23 \\
\hline
\end{tabular}

It should also be noted that none of these measured patterns was successfully fitted assuming a pure $\mathrm{Si} / \mathrm{Ge}$ multilayer, as in the nominal structures (Tab. I). In fact, a disagreement between experimental and theoretical curves was found even in the position of the zero-order satellite peak if assuming a pure Ge layer with the nominal thickness. The best agreement was obtained supposing a $\mathrm{Si}_{x} \mathrm{Ge}_{1-x} / \mathrm{Si}$ multilayer. Furthermore, a pronounced modulation of the Pendellösung fringes was measured around each satellite peak (Fig. 6). The higher order satellite peaks are strongly affected by the interface roughness and thickness variations and, consequently, can be successfully simulated only by using a statistical theoretical approach. Therefore, we limited our analysis to the asymmetry of the Pendellösung fringes around the zero-order satellite peak, the 


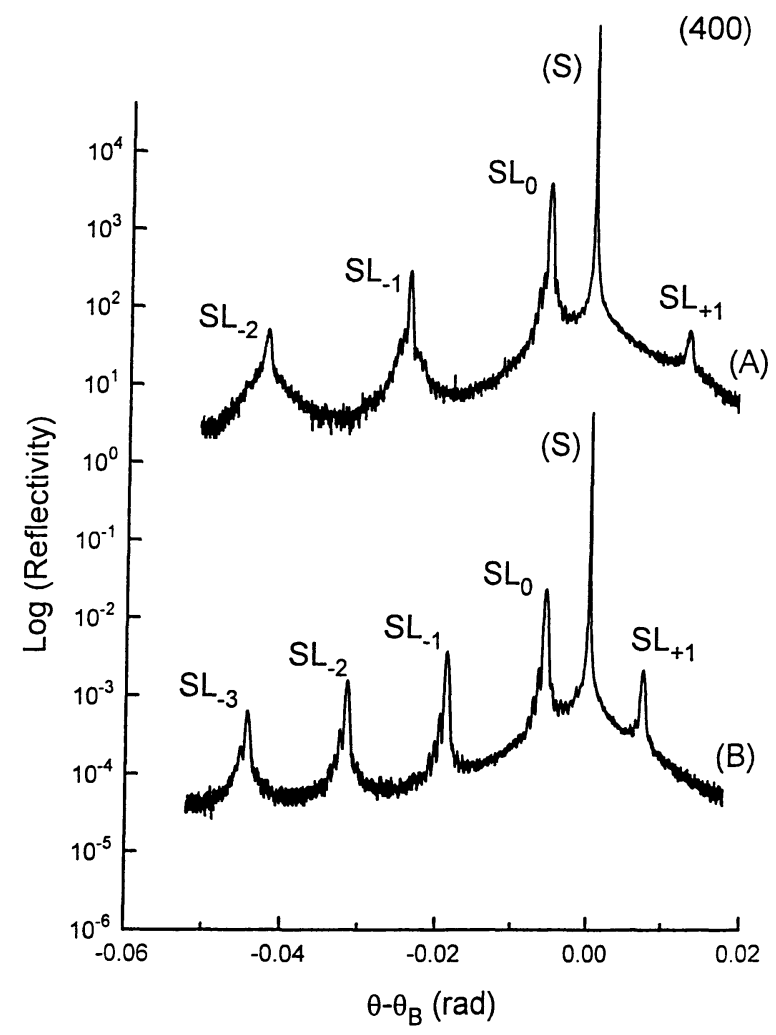

Fig. 6. - High-resolution X-ray diffraction profiles recorded in the vicinity of the Si(400) reflection. A and $B$ refer to the samples $\sharp 230$ and $\sharp 233$, respectively.

Table III. - Strain gradient $\varepsilon_{\mathrm{g}}(t)$ in the sample $\sharp 230$ as a function of the growth thickness $t$.

\begin{tabular}{|l|l|l|l|l|l|l|l|l|}
\hline$t(\mathrm{~nm})$ & $0-80$ & $80-150$ & $150-160$ & $160-161$ & $161-162$ & $162-163$ & $163-164$ & $164-165$ \\
\hline$\varepsilon_{\mathrm{g}}(\mathrm{t}) \times 10^{-3}$ & 8.350 & 8.572 & 9.977 & 10.262 & 10.398 & 10.601 & 10.771 & 10.940 \\
\hline
\end{tabular}

latter being almost no affected by the already mentioned interface problems [32]. Consequently, the asymmetry of the Pendellösung fringes around the zero-order peak is principally related to a strain gradient, i.e. of a varying chemical composition $\mathrm{Si} / \mathrm{Ge}$ ratio. In Table III the strain gradient $\left(\varepsilon_{\mathrm{g}}\right)$ used to simulate the zero order peak of our experimental measurements for sample $\sharp 230$ is reported; $\varepsilon_{\mathrm{g}}(t)$ is the value of the strain within the epilayer as a function of the thickness of the epilayer, starting from the substrate $(t=0)$ to the top of the film $(t=165 \mathrm{~nm})$. It is worthwhile to remark that the only free parameter of the X-ray simulation is the strain within the epilayer considering that the thickness of each $\mathrm{Si}_{x} \mathrm{Ge}_{1-x}$ layer is assumed from the TEM measurements. 


\section{Discussion and Conclusions}

The above results show the intermixing of $\mathrm{Ge}$ and $\mathrm{Si}$ atoms during the growth of $\mathrm{Si}_{x} \mathrm{Ge}_{1-x} / \mathrm{Si}$ multilayer [33]. Within the accuracy of the TEM measurement, no difference was detected between the thickness of the $\mathrm{Si}_{x} \mathrm{Ge}_{1-x}$ layers in the two samples. This result should be related to the diffusion length of $\mathrm{Ge}$ in $\mathrm{Si}$, in particular because the presence of strain enhances the interdiffusion process producing an intermixing of about 3-4 ML [34]. In fact, at the growth temperature of our samples, the diffusion length is about $1.1 \mathrm{~nm}[35,36]$ which agrees very well with the measured $(12 \pm 2) \mathrm{nm} \mathrm{Si}_{x} \mathrm{Ge}_{1-x}$ layers thickness. This diffusion length produces a broadening of the nominal pure Ge layer (Tab. I) resulting in a wider layer of $\mathrm{Si}_{x} \mathrm{Ge}_{1-x}$ alloy as measured from TEM micrographs. Moreover, we observe in each Ge layer of all the observed samples the quasiperiodic variation of the thickness of the Ge layer related to a defect-free Skranski-Krastanov (S-K), growth mode [15,22]. The waviness allows the sample to concentrate the strain energy in the islands reducing the strain energy density in the $\mathrm{Si}_{x} \mathrm{Ge}_{1-x}$ layers. A film with islands has a lower energy with respect to a flat, uniformly strained film. In fact, a strain-relieved island reduces the elastic energy over its volume paying a proportionally smaller cost of additional surface energy [37]. Furthermore, depending on the efficiency of the strain relaxation, a coherent island may have a lower energy than a dislocated island because of the energy cost associated with dislocation nucleation [11]. A coherently islanded film is not completely relieved since a non-uniform strain gradient exists, because the top of the film has a different strain with respect to the first layer. Consequently, at the edges of the island the strain-energy density is higher, and in these sites homogeneous nucleation of dislocations has a higher probability. We define by " $y$ " the ratio between the height of the coherent islands and its width. As the strain energy increases, the $y$-ratio increases until the amount of energy for the nucleation of dislocations becomes very small and the strain energy is relieved creating the first nucleus of the "conical shaped defect" (Fig. 2 to Fig. 5). During the MBE growth the remaining part of the epilayer is influenced by this process, but the areas far from the "conical shaped defects" will continue to grow epitaxially. As seen in Figure 2 and Figure 5, the extended defects nucleate far from the first substrate-epilayer interface. Consequently the origin of these defects is due to the achieving of the critical thickness of the relevant period and not of the multilayer as a whole. The increasing of the strain energy (Tab. III) in the relevant period, measured from the X-ray patterns, should be due to a monotonic increase of Ge content as a function of the growth time. This increase in the Ge content could be due to an anisotropic diffusion of $\mathrm{Ge}$ atoms in the Si layer.

The results obtained from our experiments on $\mathrm{Si}_{x} \mathrm{Ge}_{1-x} / \mathrm{Si}$ multilayer can be summarized as follows:

i) an inter-diffusion of Si-Ge in each period of all the observed specimens has been observed and it has been correlated to the inter-diffusion length at the specific growth temperature;

ii) the strain in each $\mathrm{Si}_{x} \mathrm{Ge}_{1-x}$ layer is partially relieved by the creation of coherent $\mathrm{Si}_{x} \mathrm{Ge}_{1-x}$ islands;

iii) the strain energy density in each $\mathrm{Si}_{x} \mathrm{Ge}_{1-x}$ layer of the multilayer increases as a function of the growth time;

iv) the coherence of the islands is preserved up to a critical value of the strain energy density and than relieved by nucleation of dislocations and creation of the so-called "conical shaped defects";

\section{Acknowledgements}

The authors would like to thank Emanuela Pesce for the skill TEM sample preparation. 


\section{References}

[1] Matthews J.W. and Blakeslee A.E., J. Cryst. Growth 27 (1974) 118-125.

[2] Matthews J.W. and Blakeslee A.E., J. Cryst. Growth 29 (1975) 273-280.

[3] Matthews J.W. and Blakeslee A.E., J. Cryst. Growth 32 (1976) 265-273.

[4] Alexander H., J. Phys. Colloq France 40(1979) C6-1-C6-6.

[5] Marée P.M., Barbour J.C. and van der Veen J.F., Kavanagh K.L., Bulle C.W.T. and Viegers M.P.A., J. Appl. Phys. 62 (1987) 4413.

[6] Hull R. and Bean J.C., Phys. Status Solidi (a) 138 (1993) 533-546.

[7] Maeda K. and Yamashita Y., Phys. Status Solidi (a) 138 (1993) 523-532.

[8] Fisher A., Kuhne H. and Richter H., Phys. Rev. Lett. 73 (1994) 2712-2715.

[9] Kasper E., in Physics and Applications of Quantum Wells and Superlattices, E. E. Mendez and K. von Klitzing Eds. (Plenum Press, N. Y. and London, 1987).

[10] People R. and Bean J.C., Appl. Phys. Lett. 47 (1985) 322.

[11] Snyder C.W., Orr B.G., Kessler D. and Sander L.M., Phys. Rev. Lett. 66 (1991) 3032-3035.

[12] Snyder C.W., Mansfield J.M. and Orr B.G., Phys. Rev. B 46 (1992) 9551-9554.

[13] Tersoff J. and Tromp R.M., Phys. Rev. Lett. 70 (1993) 2787-2785.

[14] Biagini M. and Catellani A., J. Appl. Phys. 76 (1994) 3516-3519.

[15] Eaglesham D.J. and Cerullo M., Phys. Rev. Lett. 64 (1990) 1943-1946.

[16] Stenkamp D. and Jäger W., Ultramicrosc. 50 (1993) 321-354.

[17] Hull R., Gibson J.M. and Bean J.C., Appl. Phys. Lett. 46 (1985) 179.

[18] Ourmazd A., Baumann F.H., Bode M. and Kim Y., Ultramicrosc. 34 (1990) 237-255.

[19] Matsuhata H., Miki K., Sakamoto K., Sakamoto T. and Yoshida S., Phys. Rev. B 47 (1993) 474-483.

[20] Rez P., Ultramicrosc. 41 (1992) 115-120.

[21] Treacy M.M.J., Gibson J.M. and Howie A., Philos. Mag. A 51 (1985) 389-417M.

[22] Gibson J.M. and Treacy M.M.J., J. Vac. Sci. Technol. B 4 (1986) 1458-1466.

[23] Perovic D.D. and Weatherly G.C. and Houghton D.C., Philos. Mag. A 64 (1991) 1-28.

[24] De Caro L., Giuffrida A.,Carlino E. and Tapfer L., Microsc. Microanal. Microstruct. 6 (1995) 465.

[25] Bierwolf R., Hohenstein M., Phillipp F., Brandt O., Crook G.E. and Ploog K., Ultramicrosc. 49 (1993) 273-285.

[26] Gibson J.M. and Treacy M.M.J., Ultramicrosc. 14 (1984) 345-350.

[27] Spence J.C.H., Experimental High Resolution Electron Microscopy (Claredon, Oxford, 1981)

[28] Shih W.C. and Stobbs W.M., Ultramicrosc. 35 (1991) 197-215.

[29] Servidori M., Centali F., Fabbri R. and Zani A., J. Appl. Cryst. 25 (1992) 46.

[30] Tapfer L., Physica Scripta 25 (1989) 45.

[31] Le Goues F.K., Copel M. and Tromp R.M., Phys. Rev. B 42 (1990) 11690-11700.

[32] Fewster P., J. Appl. Cryst. 21 (1988) 524.

[33] Copel M., Reuter M.C., Horn Von Hoegen M. and Tromp R.M., Phys. Rev. B 42 (1990) 11682-11689.

[34] Iyer S.S., Tsang J.C., Copel M.W., Pukite P.R. and Tromp R.M., Appl. Phys. Lett. 54 (1989) 219-221.

[35] Fukatsu S., Fujita K., Yamaguchi H., Shiraki Y. and Ito R., App. Phys. Lett. 59 (1991) 2103.

[36] Fujita K., Fukatsu S., Yamaguchi H., Igarashi T., Shiraki Y. and Ito R., Jpn. J. App. Phys. 29 (1990) L1981.

[37] Luryi S. and Suhir E. App. Phys. Lett. 49(1986) 140-142. 